\title{
Gestión educativa y el desempeño docente en las instituciones educativas de Castilla, 2015
}

\author{
Educational management and teaching performance in \\ educational institutions in Castilla, 2015
}

\section{- CLAUDIA DIVANI MAZA SÓCOLA}

divanimaza@gmail.com

Código ORCID: 0000-0001-9882-9498

Universidad César Vallejo, Perú

Artículo recibido en octubre 2020 / Arbitrado en noviembre 2020 / Publicado en enero 2021

Resumen

Palabras clave:

Abstract

Keywords:
La presente investigación tuvo como propósito determinar la relación que existe entre la gestión educativa y el desempeño docente de las Instituciones Educativas del Distrito de Castilla en el año 2015. El enfoque utilizado fue cuantitativo, con un diseño no experimental, descriptivo y correlacional. La población estuvo constituida por 88 docentes de cuatro instituciones educativas de Castilla del nivel de Inicial, y la muestra, al ser la población pequeña, incluye a toda la población. La técnica que se empleó fue la encuesta y el instrumento el cuestionario tipo Likert. Entre las principales conclusiones del estudio se puede mencionar una gestión educativa regular, caracterizada por una gestión organizacional, pedagógica-didáctica, comunitaria y administrativa también regular. El desempeño docente de las instituciones investigadas está en un nivel medio, producto del nivel medio en el desempeño pedagógico, político y cultural. El estudio indica que la gestión educativa se relaciona significativamente con el desempeño docente.

Gestión Educativa, desempeño docente, gestión pedagógica-didáctica, gestión cultural y gestión política

The purpose of this research was to determine the relationship that exists between educational management and teaching performance of the Educational Institutions of the Castilla District in 2015. The approach used was quantitative, with a non-experimental, descriptive and correlational design. The population consisted of 88 teachers from four elementary school level educational institutions in Castilla, and the sample, being the small population, includes the entire population. The technique used was the survey and the instrument the Likert-type questionnaire. Among the main conclusions of the study, we can mention a regular educational management, characterized by an organizational, pedagogical-didactic, community and administrative management that is also regular. The teaching performance of the investigated institutions is at a medium level, a product of the medium level in pedagogical, political and cultural performance. The study indicates that educational management is significantly related to teaching performance.

Educational Management; teaching performance; pedagogical-didactic management; cultural management and political management 


\section{INTRODUCCIÓN}

Uno de los mayores trabajos de los grupos sociales educativos es la necesidad de que sus acciones perduren el tiempo, en medio de un mundo cada día más exigente y globalizado, lo que ha obligado a las Instituciones educativas a realizar cambios en sus estructuras, orientados a mejorar la calidad de sus procesos. En este sentido, la gestión educativa, se ha convertido en una herramienta útil para lograr la mejora del desempeño docente, en la búsqueda por alcanzar el logro de sus objetivos. Se entiende que la gestión educativa es el motor de las instituciones educativas; es la encargada de motivar y dinamizar las actividades al interno de la institución y de manera particular al desempeño docente. Teniendo en cuenta que el capital más importante de las instituciones educativas es su personal, su desempeño y sobre todo la práctica docente se convierte en pilar fundamental del éxito de la institución educativa.

Desde esta perspectiva la gestión no solo implica un conjunto de operaciones destinadas a resolver alguna situación problemática en una organización sino también ayuda al logro de los objetivos y metas institucionales. Estas acciones además están integradas de manera que se puedan alcanzar los objetivos en el corto, mediano y largo plazo. Dichas acciones sirven de enlace entre la planificación y los objetivos que se desean alcanzar. Es todo un proceso que se articula con el equipo directivo docente; se elabora entorno a los objetivos y metas institucionales, los cuales están ligados a objetivos nacionales. Es un proceso que se reproduce en forma cíclica, que se va renovando o modificando en cuanto se van cumpliendo las metas y alcanzando los objetivos.
En relación con la temática planteada en torno a la importancia de la Gerencia Educativa, se considera relevante mencionar los antecedentes que sustentan este estudio. Tal es el caso de la publicación de la Oficina Regional de Educación de la UNESCO (citada por Quichca, 2012), donde se afirma que el concepto de excelencia y calidad de la gestión educativa se refleja en la calidad de la educación y se levanta como un problema en América Latina y en Chile desde principio los 90. Es a partir de esa fecha que el concepto de calidad se convierte en un tema de investigación prioritario en la mayoría de proyectos de educación de los países de América Latina y el Caribe y además, es incluida entre los objetivos fundamentales del Proyecto Principal de Educación de la UNESCO.

Continuando con los antecedentes, dentro de los estudios revisados a nivel internacional se han tomado aquellos que están estrechamente relacionados con la investigación; así tenemos a Robayo y Bojacá (2009), quiénes en su estudio denominado "Propuesta de Gestión Educativa de la ética del cuidado de sí" ponen énfasis en la aplicación de las técnicas de descripción, clasificación y explicación. Se trata de un estudio realizado en la ciudad de Bogotá, y está basado en una muestra 118 estudiantes, 18 padres de familia, 14 docentes y los administrativos de ambas jornadas. El estudio concluye en afirmar que el trabajo de una buena gestión, seria, coordinada y organizada, permite que las cosas funcionen de manera eficiente. Asimismo, permite el logro de metas establecidas a corto, mediano y largo plazo. Este estudio aporta a la investigación nuevos enfoques teóricos que valoran la importancia que tiene la gestión educativa para el logro de las metas propuestas, así como también para 
el logro de las tareas educativas reflejadas en el desempeño docente.

Otro aspecto relevante entre estos antecedentes está en Yabar (2013) y su tesis "La Gestión Educativa y su relación con la Práctica Docente en la Institución Educativa Privada Santa Isabel de Hungría de la ciudad de Lima Cercado". En esta investigación se señala que el concepto de gestión es totalmente dinamizador de los diferentes insumos que intervienen en las unidades educativas. El estudio encontró que la gestión educativa, la evaluación y el planeamiento juegan un rol fundamental en la práctica docente, evidenciando una relación directa entre ellos. También concluye que hay una relación directa entre la gestión, la ejecución y el control de la gerencia educativa con práctica docente. La investigación se relaciona con la presente, al resaltar que la gestión educativa pretende responder a las necesidades reales y ser un ente motivador $y$ dinamizador interno de las actividades educativas.

En este orden de ideas, ya que el capital más importante lo constituyen los principales actores educativos, es fundamental que éstos cuenten con una gestión eficiente que permita que los docentes unan sus esfuerzos para mejorar la práctica del día a día, tomando en cuenta los aspectos relevantes, las expresiones, el reconocimiento de su contexto y las principales situaciones a las que se enfrentan.

Por lo visto anteriormente se puede decir que el estudio de los antecedentes pone en evidencia el interés de los investigadores por la gestión educativa y por el desempeño docente; sin embargo, son pocos los trabajos que relacionan ambas variables; la mayoría de antecedentes evalúa cada variable por separado. En el caso de los estudios correlaciónales, se encontraron antecedentes que relacionan la gestión docente con la función docente; la calidad de la formación profesional con la práctica docente y un solo antecedente trata el tema de la relación con el desempeño docente.

Esta falta de conocimientos sobre la forma en que se relacionan las variables en el ámbito local, ha motivado el presente estudio; es más, ante la carencia de instrumentos de recolección de datos estandarizados, se han construido instrumentos propios, los que pueden servir como referencia para otras investigaciones a desarrollarse en contextos similares.

Partiendo de lo expuesto anteriormente, es posible decir que uno de los grandes problemas que enfrentan las instituciones educativas del siglo $\mathrm{XXI}$, está relacionado con la falta de políticas y acciones educativas que propicien la creación de espacios adecuados para que el docente ejerza en forma articulada y proporcionada, las funciones académicas que le competen a su ejercicio profesional. En consecuencia, el desempeño docente no se concibe como una unidad compleja, integrada, sino como un conjunto de conocimientos parcelados, es por ello que diversos países han promovido, con este propósito, la definición de criterios de un adecuado desempeño docente para que sea tomado en cuenta como un primer peldaño en el proceso de construcción e implementación de políticas públicas dirigidas a fortalecer la profesión.

Es de recalcar que la gestión educativa alude también a un cierto comportamiento organizacional, caracterizado por la movilización de toda la comunidad educativa en donde el desempeño de los docentes involucrados, es el de tomar una posición frente al entorno del campo educativo. Como se puede observar, el 
problema fundamental del sistema educativo es la calidad. Siendo el profesor uno de los elementos más importantes de la tarea educativa. Hablar actualmente del desempeño docente es una noción que ha cobrado gran relevancia en las discusiones internacionales sobre la función de los maestros en el éxito educativo. Se viene hablando del desempeño docente a principios del año 2000, en América Latina, en donde se reconoce a los docentes como actores protagónicos del cambio educativo.

En el contexto actual, el desempeño docente refleja una urgente necesidad de contar con criterios consensuados que orienten los buenos procesos de evaluación y de servicio de los maestros peruanos, quienes deben responder a una realidad problemática, promoviendo el desarrollo personal y profesional, el cual debe estar acompañado de una buena gestión educativa, es decir, aquella que cree condiciones, que construya escenarios adecuados, y que promueva el buen desempeño de los docentes.

Actualmente esto no se visualiza ni se percibe en muchas de las instituciones investigadas. La mayoría tienen deficiencias en la gestión educativa, lo que les impide abordar los agentes que están directamente relacionados con los alumnos y sus logros para el éxito. Ocurre igual con el resto una comunidad educativa que exige cada vez más. Por esta razón, los docentes deben atender a un mayor compromiso en el desempeño laboral.

En torno a la problemática planteada surge la siguiente interrogante de investigación: ¿Cuál es la relación de la gestión educativa y el desempeño docente en las Instituciones Educativas del distrito de Castilla, 2015? De esta pregunta se deriva el siguiente Objetivo
General: Determinar la relación que existe entre la gestión educativa y el desempeño docente de las instituciones educativas del distrito de Castilla en el año 2015. Para llegar lograr dicho objetivo general fue necesario pasar por los siguientes objetivos específicos:

1. Identificar el nivel de las dimensiones de las variables gestión educativa $y$ desempeño docente de las instituciones educativas del distrito de Castilla en el año 2015.

2. Establecer la relación entre las dimensiones organizacional, pedagógico-didáctico, comunitaria y administrativa con el desempeño docente en las IE del distrito de Castilla en el año 2015.

Continuando con la línea metodológica, la presente investigación se justifica mediante los siguientes aspectos: permite determinar la relación de la Gestión Educativa con el desempeño docente. En lo teórico se ha trabajado con los planteamientos de Cassasus (2000), quien señala que la gestión educativa no es solamente pragmática como podría pensarse, sino que la dimensión política está inscrita en su práctica y la gestión de la escuela está imbricada dentro las políticas educativas que se despliegan desde el Estado como políticas públicas.

Así pues, esta teoría se centra en que la gestión educativa hace referencia a la acción y el pensar de los educadores en situaciones concretas, de las cuáles también es necesario distanciarse y representarse la realidad simbólicamente para reflexionarla, analizarla, expresarla y denunciarla. Es necesario considerar que la gestión educativa posee en sí misma, una complejidad que incluye las 
subjetividades de las personas, la realidad institucional y fundamentalmente, el entorno. Es decir, para gestionar se requiere del conocimiento sensible como actitud frente a la realidad. Desde esta perspectiva toda apreciación, definición o conceptualización acerca de la gestión educativa acarrea una posición frente a la realidad. Por esto se requiere repensar la gestión educativa desde la propia situación local dado que, aun teniendo elementos teóricos valiosos, no se adapta en muchos casos a la propia realidad.

Otro aporte de esta investigación el análisis que hace del desempeño docente fundamentado con el Marco de Buen Desempeño Docente (2012). Para dicho estudio, el desempeño del docente se deriva del análisis de sus dimensiones: pedagógica, cultural y política. También se contó con aportes de Díaz (2004) cuyo estudio permite entender el comportamiento de los docentes, incluyendo procesos de gestión para transformar el papel que desempeña la escuela y el logro de los objetivos trazados como institución.

Por lo tanto, la presente investigación aporta nuevos cimientos y teorías que coinciden en señalar la importancia de una buena gestión en los colegios e instituciones educativas de Castilla. Se considera esta problemática, como un distanciamiento entre las personas que llevan a cabo la gestión de las instituciones y sus docentes.

Cabe destacar que el contexto elegido para esta investigación está inmerso en un desinterés educativo y pedagógico, produciendo falta de motivación en la labor que desempeñan los docentes. Se puede decir que los esfuerzos de las instancias responsables para una buena gestión educativa no son suficientes. Analizando esta mirada en las Instituciones Educativas de Castilla, se propone impulsar los diseños de estrategias de Gestión Educativa, teniendo en cuenta los diversos estudios o teorías en los que se basa la investigación. Al identificar los actores de esta problemática se evidencia que está en manos de la Gestión Educativa motivar a implementar y desarrollar estrategias educativas de gestión, invitando a la participación activa de directivos, docentes y comunidad y promover su crecimiento y desarrollo.

\section{MÉTODO}

En cuanto al aspecto metodológico, la presente investigación estuvo orientada a estudiar dos variables: La Gestión educativa y El desempeño docente y su posible relación en un contexto de la práctica educativa.

Para comprender las variables de la investigación y su posible relación, el estudio se fundamentó en el paradigma cuantitativo porque este permite obtener información empírica de los hechos, utilizar métodos estadísticos para el análisis e interpretación de datos y contrastar hipótesis de investigación.

El problema se aborda utilizando una investigación no experimental, de tipo descriptiva correlacional porque en el estudio, el investigador recoge información de la gestión educativa y del desempeño docente, tal y como se manifiesta en los sujetos investigados sin ninguna intervención. El estudio es descriptivo correlacional porque en primer lugar se caracteriza cada variable y luego se estudia su relación. La población estuvo conformada por los docentes de las Instituciones educativas de Castilla, detalladas a continuación en la tabla 1. 
Tabla 1. Conformación de la población y la muestra

\begin{tabular}{lcc}
\hline INSTITUCIÓN & POBLACIÓN & MUESTRA \\
\hline IEFAP "Samuel Ordoñez Velásquez" & 50 & 50 \\
IEI "015" & 20 & 20 \\
IEI "Caritas felices" & 10 & 10 \\
IEI "Mundo de colores" & 8 & 8 \\
\hline
\end{tabular}

Como la población en cada uno de los colegios es pequeña y está al alcance del investigador, la muestra coincide con la población. Al tomarse como muestra a todos los elementos de la población, no se utilizó ningún tipo de muestreo.

En cuanto a la técnica de recolección de datos, se empleó un documento que contiene preguntas relacionadas a cada uno de los indicadores de las variables conocido como encuesta. En concordancia con dicha técnica, se utilizó como instrumento un cuestionario estructurado en escalas de Likert. Este fue validado y evaluado en su confiabilidad mediante el "Juicio de expertos", los cuales permitieron elaborar el instrumento final. La confiabilidad del instrumento se logró utilizando el índice de consistencia y coherencia interna Alfa de Cronbach, cuyo valor fue de 0.896 para el cuestionario de gestión educativa y de 0.738 para el desempeño docente, lo que confirma la confiabilidad del instrumento.

Luego de analizada la información, ésta se sistematizó en el programa estadístico IBM SPSS 22.0, en el cuál se realizó todo el análisis estadístico. Los resultados se presentaron en tablas con frecuencias absolutas y porcentuales. Para contrastar las hipótesis de investigación se utilizó el coeficiente de correlación de Spearman, debido a que las variables y sus dimensiones se construyen a partir de preguntas medidas en escala ordinal.

\section{RESULTADOS}

A continuación, se presentan los resultados obtenidos durante el estudio una vez aplicado el análisis de las variables. El mismo se llevó a cabo según el nivel de cada dimensión, utilizando para ello la percepción media de los encuestados por las preguntas que conforman dicha dimensión.

De este modo, en cuanto a ítem número 1 , referente al nivel alcanzado en la gestión educativa, los resultados del estudio indican que en general la gestión educativa en las instituciones del distrito de Castilla en el año 2015 , fue regular, según lo indica el $80.7 \%$ de encuestados. Sólo el $12.5 \%$ lo considera como buena, y el resto, $6.8 \%$, lo califica como deficiente. Con esto se evidencia que esta es básicamente una gestión organizacional, pedagógica-didáctica, comunitaria y administrativa regular, según el $62.5 \%, 56.8 \%$, $69.3 \%$ y $50 \%$ de docentes. El estudio también indica que hay un porcentaje significativo de docentes, $19.3 \%$ y $37.5 \%$, que califican a la gestión organizacional y administrativa como deficiente. Estos resultados se pueden apreciar en la figura 1. 


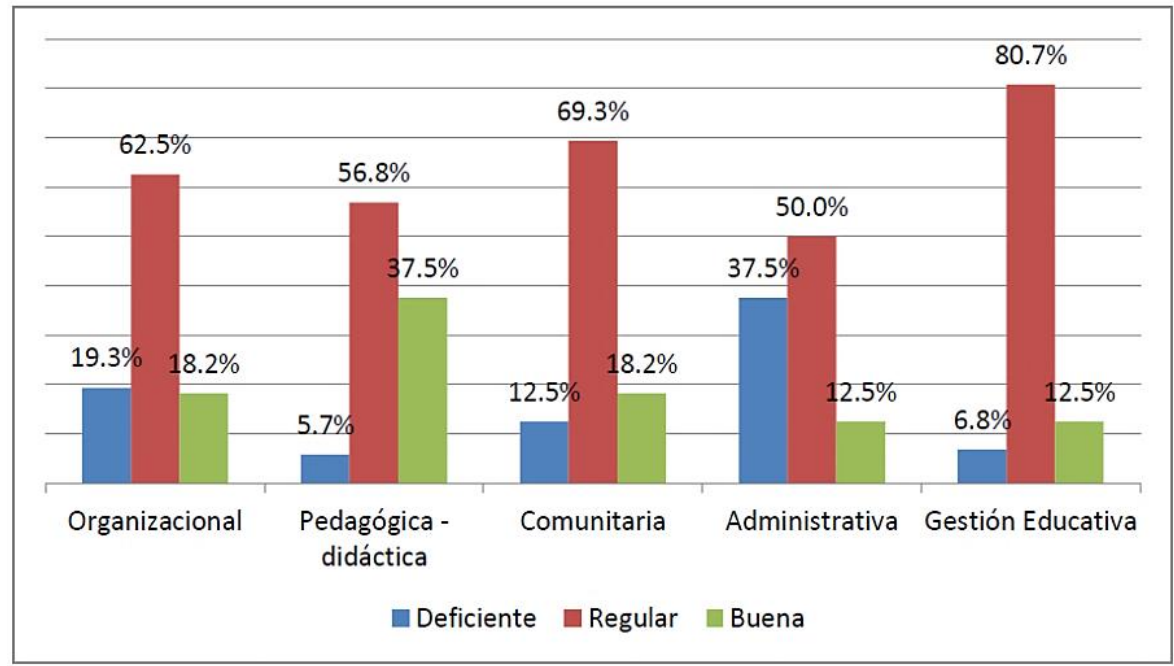

Figura 1. Nivel de la Gestión Educativa

De acuerdo a los resultados del segundo ítem, el desempeño docente es calificado mayoritariamente como regular, según lo indica el $81.8 \%$ de los encuestados. Este se caracteriza fundamentalmente por un desempeño pedagógico, cultural y político calificados como regulares, como se observa en el 75\%, $62.5 \%$ y $81.8 \%$ respectivamente. En el caso de los aspectos culturales, los resultados indican que hay una cifra importante ya que un $25 \%$ afirman que su gestión es deficiente. Ver figura 2.



Figura 2. Nivel de desempeño docente 
Seguidamente, los resultados a continuación en la figura 3, muestran que el desempeño medio se refleja mayormente en docentes que califican a la gestión organizacional como deficiente, en cambio el buen desempeño se hace presenta cuando la gestión organizacional es buena. Estos resultados evidencian que el desempeño docente mejora al mejorar la gestión organizacional.

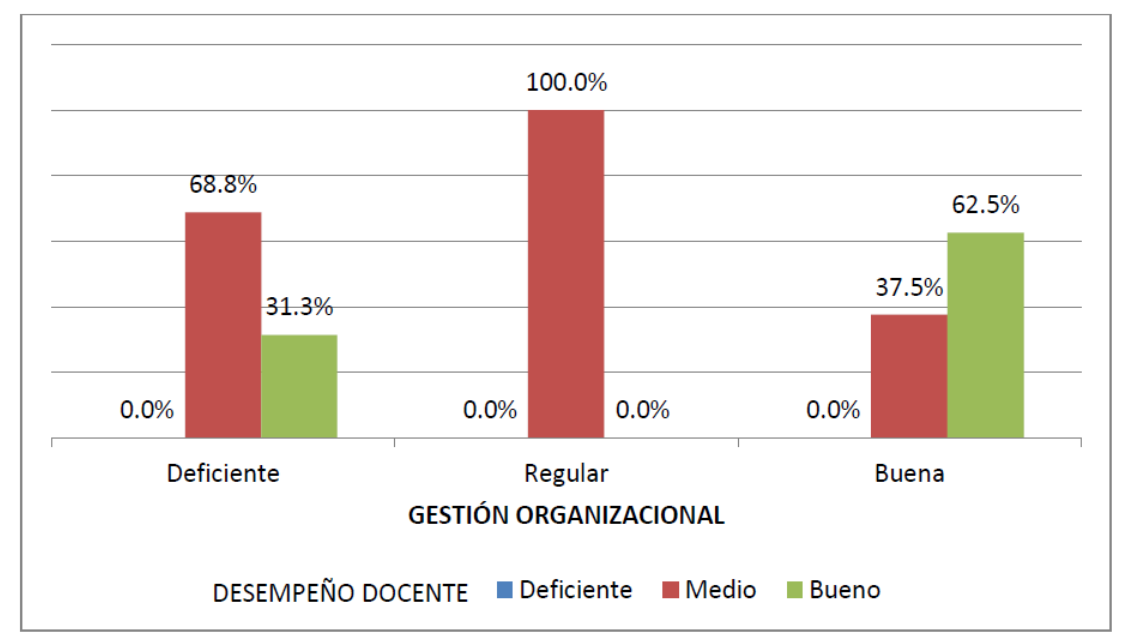

Figura 3. Relación entre la gestión organizacional y el desempeño docente

En la figura 4 que se muestra a continuación, se evidencia que los docentes que califican al desempeño como regular, son aquellos que consideran que la gestión pedagógica didáctica es deficiente; en cambio el buen desempeño docente se manifiesta más cuando dicha gestión es calificada como buena. Estos resultados demuestran una tendencia de que al mejorar la gestión pedagógica-didáctica, también mejora el desempeño docente.

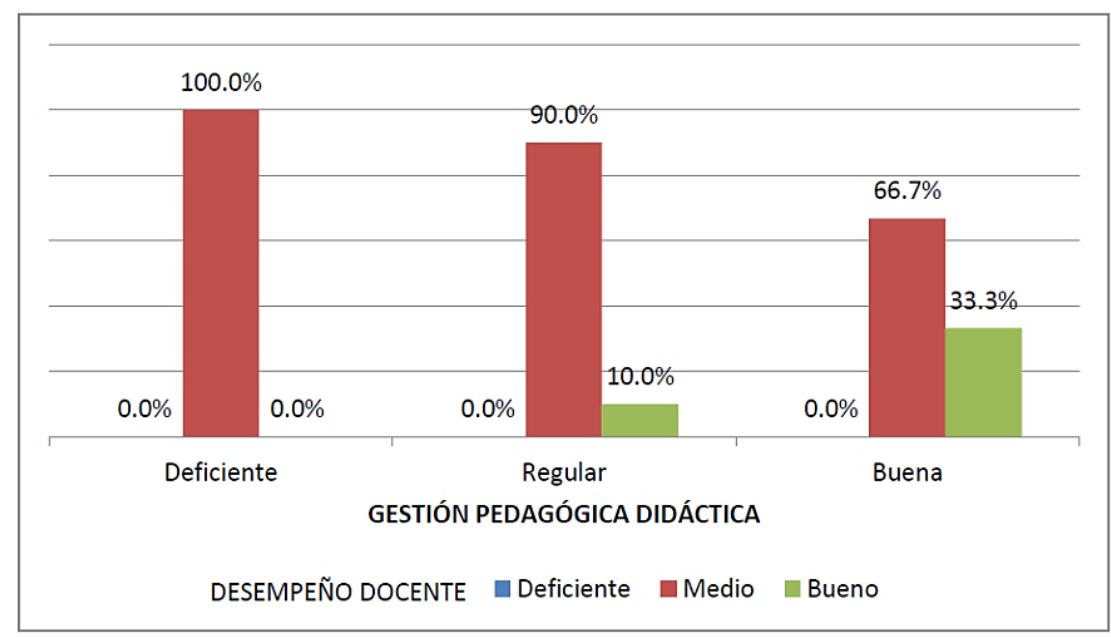

Figura 4. Relación entre la gestión pedagógica-didáctica y el desempeño docente 
Los resultados muestran también que el buen desempeño docente a nivel medio o regular, se manifiesta en los encuestados que califican a la gestión comunitaria como deficiente; mientras que, aquellos que evidencian un buen desempeño son los que califican a la gestión comunitaria como regular o buena. Los resultados dejan en evidencia que hay cierta tendencia a mejorar el desempeño, en la medida que mejora la gestión comunitaria. (Figura 5)



Figura 5. Relación entre la gestión comunitaria y el desempeño docente

De este modo, de acuerdo con los resultados de la gestión administrativa, es calificada como deficiente, el desempeño docente es básicamente medio, mientras que cuando la gestión es considerada buena, el desempeño también es bueno. En este caso también se nota cierta tendencia a que el desempeño mejora en tanto mejora la gestión administrativa. (Figura 6)

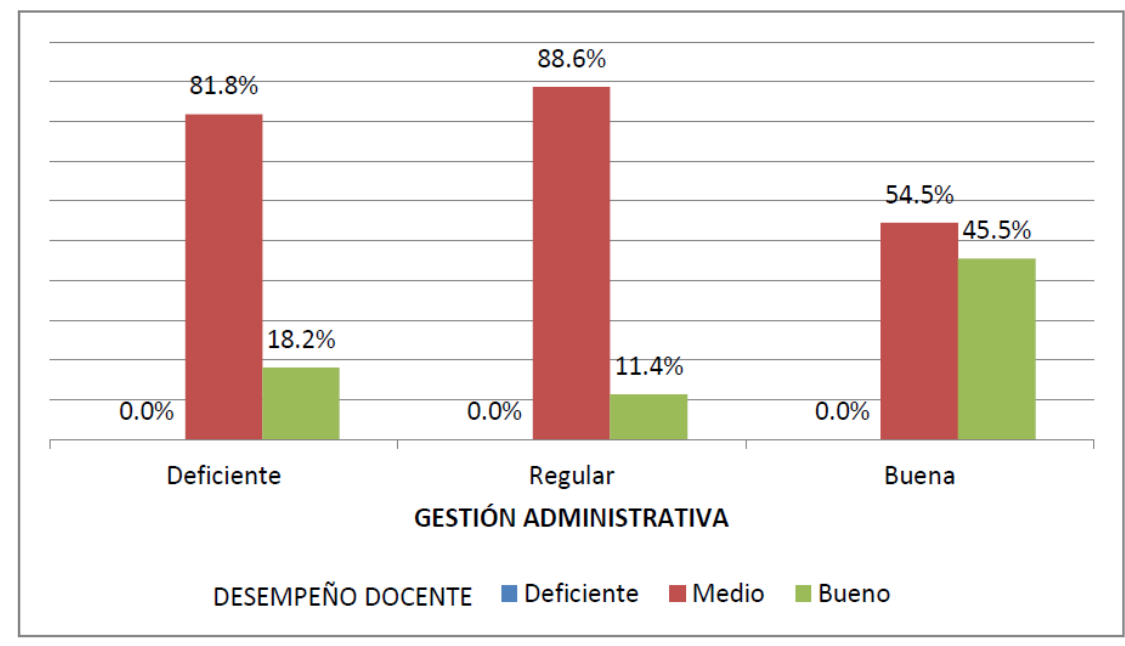

Figura 6. Relación entre la gestión administrativa y el desempeño docente 
Seguidamente, los resultados a continuación en la figura 7 , dejan en evidencia que el desempeño medio de los docentes se manifiesta en presencia de una gestión deficiente, en tanto que el buen desempeño, aparece cuando la gestión educativa es también es buena. Este resultado deja en claro que el desempeño docente tiende a ser mejor, en la medida que la gestión educativa también mejora.

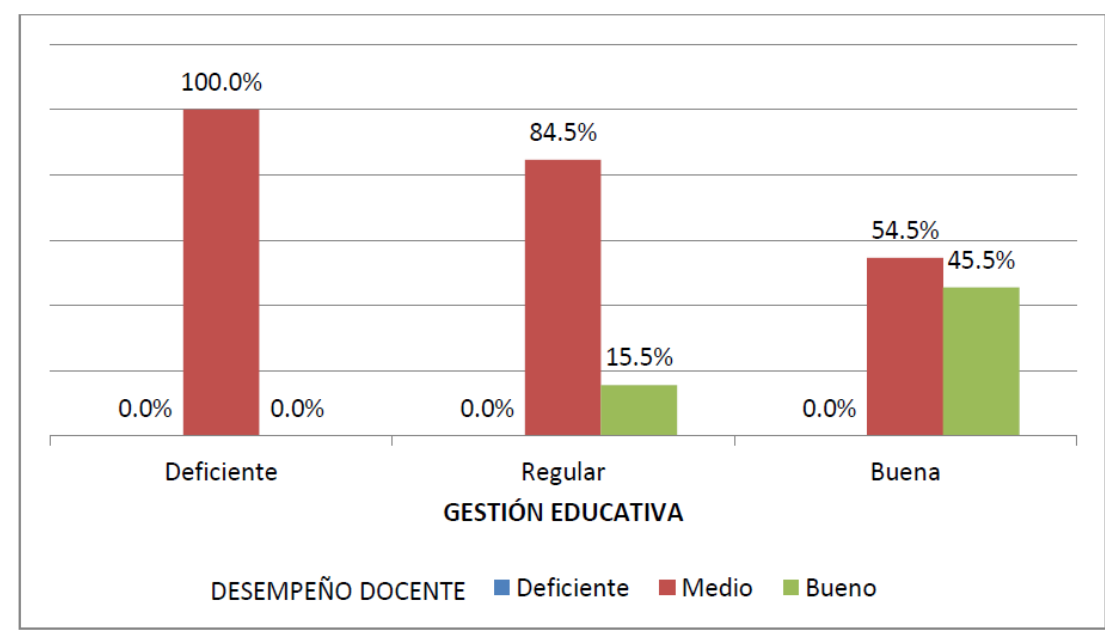

Figura 7. Relación entre la gestión educativa y el desempeño docente

DISCUSIÓN

Los resultados de la aplicación de los cuestionarios, se discuten a continuación teniendo en cuenta los objetivos de la investigación. El primer objetivo específico estudia cómo es la gestión educativa y el desempeño docente en las IE del distrito de Castilla.

Sobre la gestión educativa existen diferentes definiciones, aunque la mayoría de autores coincide con Casassus (2000), quién señala que "es la acción humana, cuyo aspecto central es la movilización de los recursos y la capacidad de generar una articulación de éstos para lograr lo que se desea" (p.4). Esta definición deja en claro la importancia que tienen los encargados de la gestión para el logro de los objetivos institucionales; los resultados de la investigación (Figura 1) evidencian que en la población, no se ha logrado una buena gestión educativa, la mayoría (80.7\%) lo califica como regular. Igualmente ocurre en la gestión organizacional (62.5\%), pedagógica-didáctica (56.8\%), comunitaria (69.3\%) y administrativa (50.0\%) calificadas también como regular. Los gestores de dichas instituciones, no han logrado integrar de manera coherente los aspectos ligados a estas dimensiones, incluso hay algunas instituciones educativas que evidencian una gestión organizacional y administrativa deficiente (6.8\%).

Por lo tanto, éstas no han logrado gestionar la disposición y organización de los recursos para obtener los resultados esperados. La gestión regular de las instituciones sujetas a investigación, no guarda coherencia con lo mencionado por Robayo y Bojacá, (2009); 
quienes indican que para que las cosas funciones bien, una gestión debe realizar un trabajo estructurado y coordinado, lo que permite alcanzar las metas establecidas por la institución educativa.

En concordancia con el objetivo mencionado al principio, la investigación está orientada también a identificar el desempeño docente de las instituciones educativas del distrito de Castilla. En cuanto a esta variable, Progré (2005) y Robalino (2005), coinciden de alguna forma en que el desempeño docente es una práctica social y cultural que ponen aplican los profesores para lograr que los estudiantes obtengan nuevos conocimientos. El estudio deja en claro que (Figura 2), los docentes no han logrado articular la parte pedagógica (75.0\%), cultural $(62.5 \% \%)$ y política $(81.8 \%), y$ en consecuencia, el desempeño docente es regular (81.8\%). Estos resultados podrían representar un serio obstáculo para aprovechar el potencial que tienen los estudiantes, como lo menciona Gutierrez (2014) el buen desempeño conduce a la eficacia docente y por lo tanto, incrementar el aprovechamiento de sus alumnos.

En cuanto a la práctica pedagógica, los docentes investigados no han logrado acumular una serie de saberes a partir de la reflexión teórica y de la práctica de la enseñanza. La dimensión cultural, que engloba conocimientos sobre el entorno donde se desarrolla el docente, tampoco es muy favorable en la mayoría de ellos. Este grupo no ha logrado satisfacer sus necesidades de conocimientos de tipo político, económico, cultural y social, lo que no les permite comprender los aprendizajes contextualizados de cada sociedad; es más, el estudio evidencia que hay un grupo importante de docentes, que solo ha logrado un desempeño mínimo esta dimensión (nivel deficiente), lo que puede representar una limitación para enfrentar los desafíos de la educación actual. En cuanto a la dimensión política, los docentes tampoco demuestran compromiso con la formación de los estudiantes como personas y ciudadanos.

Otro de los objetivos específicos estuvo orientado a determinar la relación entre la gestión organizacional y el desempeño laboral. El estudio demostró que la que la dimensión organizacional se relaciona significativamente en el desempeño docente. Según Frigeiro y Poggi (1992), la gestión organizacional es la que determina el estilo de funcionamiento de las instituciones educativas y agrupa una serie de aspectos estructurales que incluyen los roles directivos, su funcionamiento, la toma de decisiones y la manera como se conducen los equipos. La correlación encontrada deja en claro la importancia que tiene este tipo de gestión para lograr un buen desempeño docente. Los resultados indican que una buena gestión organizativa es clave para desarrollar las actividades docentes. Esta correlación encontrada permite aceptar que la gestión organizacional está significativamente relacionada con el desempeño académico. Estos aspectos repercuten positivamente en la capacidad de los docentes para participar y reflexionar en la toma de decisiones institucionales, así como en su tarea de lograr que los estudiantes alcancen las competencias esperadas.

En cuanto al objetivo que busca establecer la relación entre la dimensión comunitaria y el desempeño docente de las IE, se demostró que este tipo de gestión tiene que ver con las 
actividades que realizan los encargados de la gestión institucional y los que participan en la toma de decisiones de cada centro educativo. Estas actividades son aspectos que según los mismos investigados, repercuten positivamente en su desempeño, es decir, en su capacidad para participar y reflexionar en las decisiones educativas, logrando un alto compromiso institucional.

Por cuanto, en la dimensión administrativa y el desempeño docente de las $\mathrm{IE}_{\mathrm{E}}$ las actividades de apoyo que realizan los encargados de la gestión tienen poca influencia en el desempeño docente y el estudio da cuenta de que no existe una correlación significativa entre ambos aspectos. Esta carencia de relación se puede explicar porque esta dimensión tiene que ver con las acciones de carácter general que implementa el sistema para la puesta en marcha del proyecto educativo. No tienen mucho significado dentro del desempeño de las tareas de los docentes. Es por esta razón que, la carencia de una relación significativa entre la gestión administrativa y el desempeño docente, no permite aceptar la hipótesis de que ambos aspectos se encuentran relacionados.

En general, el estudio demuestra que si bien no todos los aspectos de la gestión educativa se relacionan con el desempeño docente, analizando de manera holística los resultados, se observa una correlación significativa entre ambos aspectos, lo que conduce a aceptar la hipótesis de que la gestión educativa se relaciona de manera significativa con el desempeño docente.

\section{CONCLUSIONES}

Retomando lo observado en los resultados anteriores es posible decir que el desempeño docente se encuentra significativamente relacionado con la gestión organizacional, este resultado implica que en la medida que dicha gestión mejore, mejorará el desempeño de los docentes.

Aunado a esto, el buen desempeño del docente se encuentra íntimamente ligado a una buena gestión pedagógica-didáctica, como lo indica la relación significativa entre ambos aspectos. De mismo modo, la gestión comunitaria está significativamente relacionada con el desempeño docente, de tal forma que si ésta mejora, los docentes también mejoraran en su desempeño. Por otra parte, cabe destacar que la gestión administrativa no evidencia una relación significativa con el desempeño docente, lo que implica que éste, tiene poco que ver con la forma como los directivos realizan la gestión administrativa.

De este modo, los resultados dejan en evidencia que el desempeño docente se encuentra directamente relacionado con la gestión educativa; este conocimiento sive como base para que las autoridades encargadas de las instituciones educativas, refuercen su gestión con el fin de mejorar el desempeño docente. Este es el aporte del estudio al conocimiento científico; es más, el estudio 
minucioso de los aspectos que se evalúan en cada variable reflejado en el cuestionario, en sí mismo requiere un nivel de análisis que va más allá de lo evidente y que es proporcionado por el nivel de estudios desarrollado por el investigador.

Es por tanto que, los directores y los consejos educativos de las Instituciones del distrito de Castilla, deben promover y organizar actividades incluidas en el Plan Anual de Trabajo, con participación de la comunidad educativa en aspectos ligados a las dimensiones: pedagógico-didáctica y administrativa, a fin de garantizar una gestión eficaz.

En este orden de ideas, se recomienda que los directivos, con el apoyo de la UGEL deben fortalecer la formación de los círculos de aprendizaje de manera mensual, para hacer intercambio de experiencias y poder mejorar el desempeño docente en los aspectos pedagógicos, políticos y culturales. Además, las IE, deben actualizar sus Reglamentos Internos y difundir oportunamente a la comunidad docente la estructura de organización, cargos, funciones, asimismo el adecuado manejo de los tiempos y recursos para mejorar la gestión.

Otro aspecto relevante en este asunto está en organizar y ejecutar talleres para los docentes tomando como base el Manual del desempeño docente y las rutas del aprendizaje, con el fin de garantizar y fortalecer la gestión pedagógica-didáctica. Por lo tanto, las IIEE deben incluir en PAT un conjunto de actividades que permitan un mayor acercamiento entre los actores educativos de la comunidad y el personal docente, a fin de mejorar cualitativamente la relación significativa observada.

De este modo, las IE deben realizar jornadas de trabajo mensuales para conocer el avance de la gestión administrativa relacionada al manejo del presupuesto institucional, monitoreo al desempeño docente, etc. para generar una gestión administrativa transparente y con resultados. Es el deber de estas IE organizar los procesos de Autoevaluación Institucional semestralmente para que la comunidad educativa reflexione $y$ proponga acciones de mejora relacionadas a la gestión educativa, incluyendo aspectos del buen desempeño docente contemplados en el Marco del Buen Desempeño Directivo.

\section{REFERENCIAS}

Casassus, J. (2000). Problemas de la gestión educativa en América Latina. (En línea) Recuperado https://silo.tips/downloadFile/problemasde-la-gestion-educativa-en-america-latinala-tension-entre-los-paradig-3

Diaz, A. (2004). Evaluación de la docencia. Su generación, su adjetivación y sus retos. ¿Es posible evaluar la docencia en la Universidad Experiencias en México, Canadá, Francia, España y Brasil? México

Frigerio, G., Poggi, M., Tiramonti, G. y Aguerrondo, I. (1992). Las instituciones educativas. Cara y ceca. Serie FLACSO. Troquel. Buenos Aires

Gutiérrez, E. (2004). Un modelo de evaluación del desempeño docente que contribuye en la mejora de la calidad de los servicios educativos. (Trabajo de Postgrado). Universidad Politécnica del Golfo de México

Pogré, P. (2005) "Desarrollo profesional y evaluación de los formadores de docentes" en Rendón Lara, Diego, Rojas García Ignacio (comp.) El desafío de formar los mejores maestros. Bogotá, Universidad Pedagógica Nacional 
Quichca, T. G. (2012). Relación entre la calidad de gestión administrativa y el desempeño docente según los estudiantes del I al VI ciclo 2010- I del Instituto superior particular La Pontificia del distrito Carmen Alto Provincia de Huamanga. Ayacucho-Perú

Robalino, M. (2005) "Una alianza para el desarrollo de los maestros", en Rendón Lara, Diego; Rojas García, Ignacio (comp.) El desafío de formar los mejores maestros. Bogotá, Universidad Pedagógica Nacional

Robayo, A. E. y Bojacá, H. (2009). Propuesta de Gestión Educativa para el colegio distrital ciudad de Villavicencio IED, desde la perspectiva de la ética del cuidado de sí. Bogotá, U Javeriana
Marco de Buen Desempeño Docente (2012). Ministerio de Educación del Perú. Resolución Ministerial Nº547-2012-ED. (En Línea) Recuperado de http://www.perueduca.pe/documents/6056 3/ce664fb7-a1dd-450d-a43dbd8cd65b4736, revisado en enero del 2016 Yabar, I. (2013). La Gestión Educativa y su relación con la Práctica Docente en la Institución Educativa Privada Santa Isabel de Hungría de la ciudad de Lima - Cercado. Trabajo de Postgrado. Universidad Nacional Mayor de San Marcos 